\title{
X-ray Surveys and Wide-Field Optical/Near-Infrared Imaging with JDEM
}

\author{
W.N. Brandt \\ Department of Astronomy 8 Astrophysics, The Pennsylvania State University, \\ 525 Davey Lab, University Park, PA 16802
}

\begin{abstract}
I briefly describe a few important scientific issues that could be addressed effectively via the combination of data from JDEM and X-ray missions. The topics covered are largely focused on active galactic nuclei (AGN) and include (1) the selection of AGN via X-ray emission and optical variability, (2) nuclear outbursts in galaxies due to transient fueling of their supermassive black holes, (3) moderate-luminosity AGN at high redshift $(z>4)$ found via application of "dropout" techniques to X-ray sources, and (4) the host-galaxy morphologies of X-ray selected AGN. I also describe the substantial challenges to obtaining wide-field X-ray data with sufficient sensitivity to complement JDEM properly.
\end{abstract}

\section{Introduction}

Extragalactic X-ray surveys have dramatically advanced over the past five years, largely due to the flood of data from the Chandra X-ray Observatory (hereafter Chandra) and the X-ray Multi-Mirror Mission-Newton (hereafter XMM-Newton). The superb X-ray mirrors and charge-coupled device (CCD) detectors on these observatories provide large source samples detected to $\approx 0.5-10 \mathrm{keV}$ flux levels that are up to 50-250 times lower than those of previous X-ray missions. They furthermore provide high-quality X-ray source positions with accuracies of $\approx 0.3-3^{\prime \prime}$, allowing reliable matching to multiwavelength counterparts. About 40 extragalactic surveys are presently underway utilizing data from Chandra and XMM-Newton.

While a broad diversity of sources are found in Chandra and XMM-Newton surveys, it is clear that the majority of the detected sources are active galactic nuclei (AGN). In fact, deep Chandra and XMM-Newton surveys have found the highest AGN sky densities to date, reaching $\approx 7,200 \mathrm{deg}^{-2}$ (e.g., Bauer et al. 2004). X-ray surveys appear to have excellent, although not perfect, 


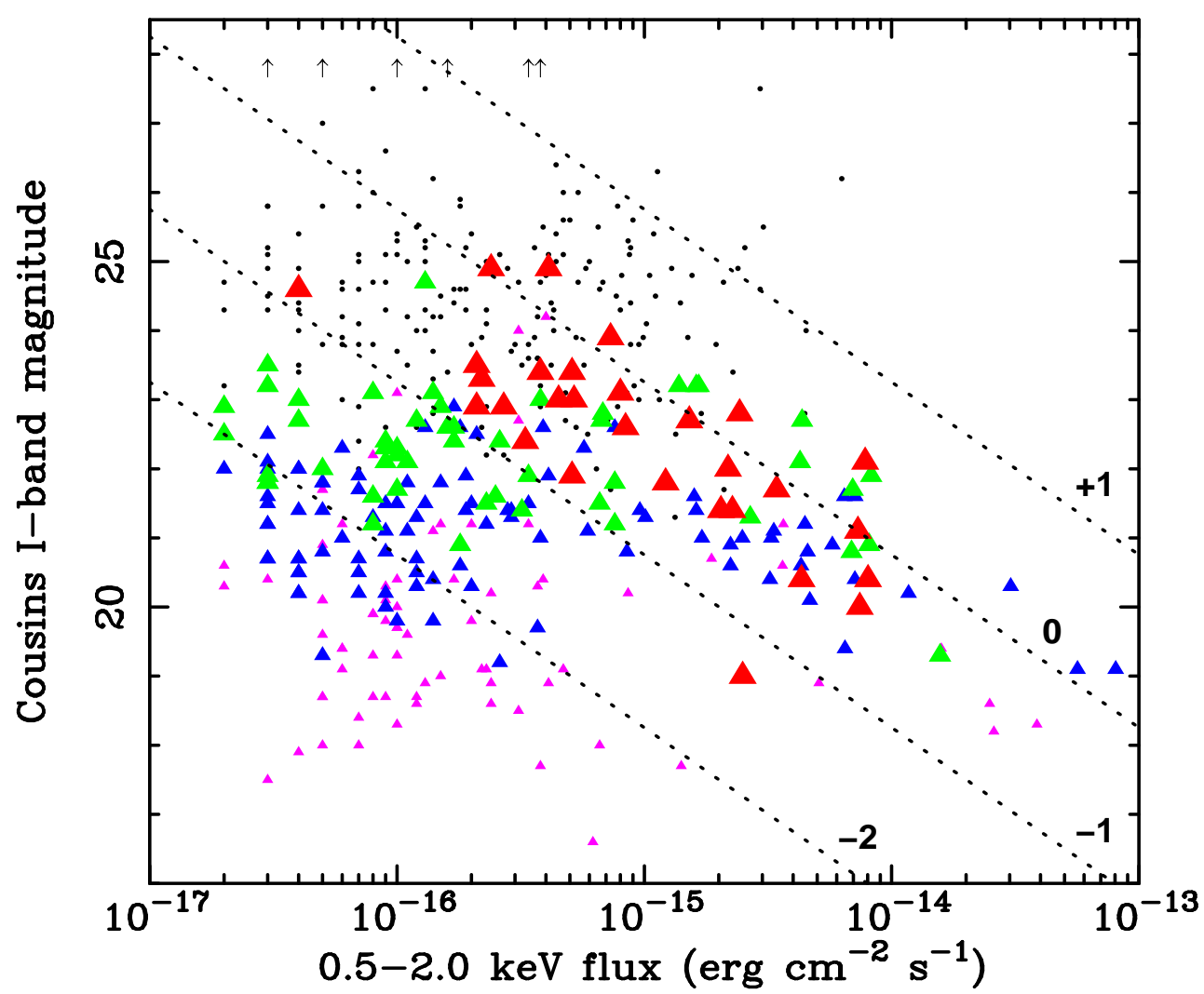

Fig. 1. I-band magnitude versus $0.5-2 \mathrm{keV}$ flux for extragalactic X-ray sources in the $2 \mathrm{Ms}$ Chandra Deep Field-North (CDF-N) observation. Sources with redshifts of $0-0.5,0.5-1,1-2$, and $2-6$ are shown as violet, blue, green, and red filled triangles, respectively (symbol sizes also increase with redshift). Small black dots indicate sources without measured redshifts. The slanted, dotted lines indicate constant values of $\log \left(f_{\mathrm{X}} / f_{\mathrm{I}}\right)$; the respective $\log \left(f_{\mathrm{X}} / f_{\mathrm{I}}\right)$ values are labeled.

completeness when compared to other multiwavelength methods for finding AGN.

Many of the AGN found in Chandra and XMM-Newton surveys are faint at optical and other wavelengths, often having $I$ magnitudes of 23-27 (see Fig. 1). They also often have modest optical luminosities so that, without superb imaging from space, there is substantial blending of the light from the AGN and its host galaxy. Thus, sensitive and high-resolution imaging at multiple wavelengths and over relatively large fields is required for optimal follow-up studies. ${ }^{1}$ Such imaging has typically lagged behind the X-ray data due to observational expense, but the Joint Dark Energy Mission (JDEM) would dramatically reverse this situation, likely leaving X-ray astronomers scrambling to "catch up."

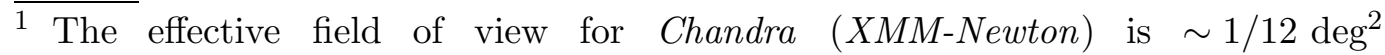
$\left(\sim 1 / 5 \operatorname{deg}^{2}\right)$, and most of the surveys mentioned above use 1-20 such fields. 
Below I will briefly describe a few exciting science projects that would be enabled by combining data from JDEM and X-ray missions such as Chandra, Constellation-X, DUO, EXIST, Generation-X, NuSTAR, XEUS, and $X M M-N e w t o n$. I will focus on AGN-driven science as AGN are the numerically dominant source population in extragalactic X-ray surveys. However, wonderful JDEM/X-ray science should also be possible for clusters and groups of galaxies, large-scale structures, starburst galaxies, normal galaxies, and other classes of objects.

\section{The Selection of AGN via X-ray Emission and Optical Variability}

Deep optical variability surveys are one of the only methods competitive with X-ray surveys at efficiently finding high sky densities of AGN. For example, Sarajedini et al. (2003) report the discovery of 16 variable galactic nuclei in the Hubble Deep Field-North (HDF-N) based on analyses of two HST V-band images taken five years apart (see Fig. 2). The derived AGN sky density is in the range $\approx 2,000-11,000 \mathrm{deg}^{-2}$. The precise sky density depends upon the amount of contamination by nuclear supernovae and statistical outliers, but it is clearly higher than that from most optical spectroscopic surveys and is plausibly comparable to that from the deepest X-ray surveys (see $§ 1$ ).

While X-ray selection and optical variability selection have respectable overlap in the AGN found (see Fig. 2), each method also finds AGN missed by the other. For example, there are at least 2-3 X-ray AGN in the HDF-N not identified by Sarajedini et al. (2003). Conversely, at least some of the variable galactic nuclei of Sarajedini et al. (2003) may be X-ray weak AGN lying below the detection threshold of even the $2 \mathrm{Ms}$ CDF-N exposure. These AGN might be X-ray weak due to a low bolometric power, or they might have emaciated accretion-disk coronae that cannot produce X-rays effectively.

As currently planned, JDEM should find at least 30,000-90,000 variability selected AGN in its $15 \mathrm{deg}^{2}$ deep survey. An even larger number of AGN should be found in its wider surveys. If appropriately matched X-ray data were obtained (see $\S 6$ ), joint JDEM and X-ray techniques could be applied to generate the largest and most complete census of moderate-luminosity, typical AGN out to high redshift. X-ray sources could be scrutinized especially carefully for optically variable nuclei in the JDEM data, and X-ray stacking techniques could be used to study the average X-ray properties of AGN not detected individually. The large solid angle coverage would allow the AGN population to be probed consistently over a wide range of luminosity; the present data have poor coverage of luminous, rare AGN. 


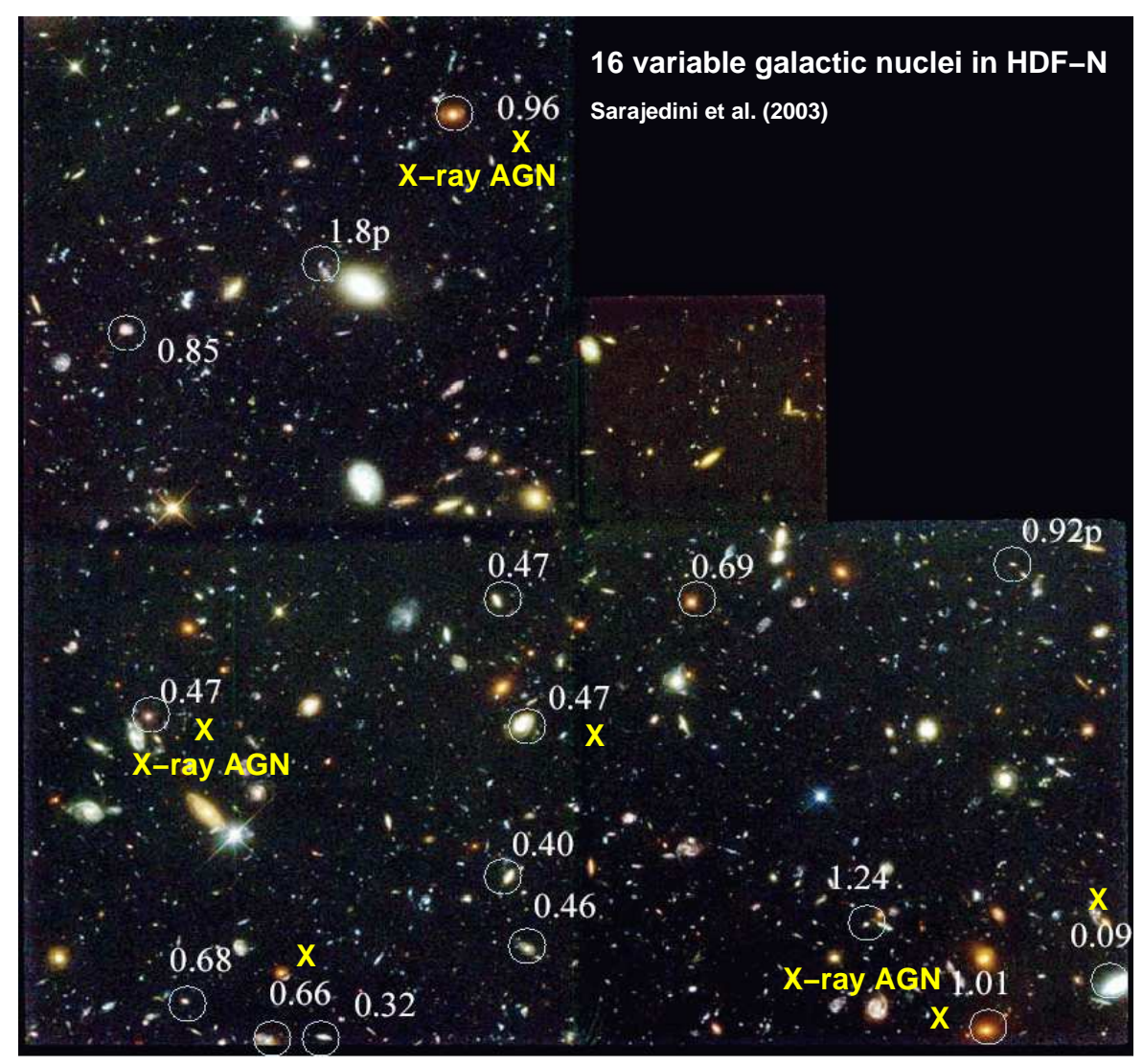

Fig. 2. HST image of the HDF-N showing the 16 galaxies with optically variable nuclei (circled and labeled with redshifts). The galaxies with both variable nuclei and X-ray detections are marked with "X" symbols; those that are clear X-ray AGN are labeled as such (there are additional X-ray AGN in the HDF-N that have not shown variable nuclei). Adapted from Alexander et al. (2003) and Sarajedini et al. (2003).

\section{Nuclear Outbursts in Galaxies}

X-ray surveys, mainly with $R O S A T$, have discovered about seven large-amplitude X-ray outbursts from galactic nuclei (e.g., Donley et al. 2002; Komossa 2002; Vaughan et al. 2004). These events have variability amplitudes of 50-400 or more, peak X-ray luminosities comparable to those of local Seyfert galaxies, soft X-ray spectra, and decay timescales of months to years (see Fig. 3a). They are observed in both inactive and active galaxies. In inactive galaxies the event rate is $\approx 10^{-5} \mathrm{yr}^{-1}$, while in active galaxies the event rate appears to be $\approx 100$ times higher. These outbursts are probably associated with transient fueling events of supermassive black holes (SMBHs). Fueling may occur when a star or planet is tidally disrupted, or in some cases it may be due to accretion-disk instabilities. 
JDEM observations should allow the discovery of additional outbursts from galactic nuclei. The X-ray outbursts above are plausibly expected to induce accompanying optical variability, and there is direct evidence for such optical variability in at least the case of IC 3599 (Brandt et al. 1995; Grupe et al. 1995). Furthermore, optical outbursts have been detected from a few galaxies without near-simultaneous X-ray coverage, including NGC 4552 (Cappellari et al. 1999) and perhaps NGC 1068 (de Vaucouleurs 1991). These outbursts should be distinguishable from nuclear supernovae based upon their spectral properties and light curves.

The current data suggest that JDEM should detect 2-20 optical outbursts from galactic nuclei in its deep, wide-field, and panoramic surveys. However, this estimate suffers from significant statistical and systematic uncertainties, and JDEM observations will allow by far the best determination of the frequency of optical outbursts throughout the Universe. Optical outbursts discovered by JDEM should be rapidly followed-up with X-ray and multiwavelength observatories to determine the nature of these events. Presently, the lack of rapid multiwavelength follow-up studies is a major hindrance to understanding.

\section{Moderate-Luminosity AGN at High Redshift}

Combined JDEM and X-ray studies should allow the discovery of hundreds of moderate-luminosity AGN at $z \approx 4-6$; only $\approx 6$ such AGN are known presently. These AGN have luminosities comparable to those of local Seyfert galaxies and low-power quasars, and at $z \approx 4-6$ their space density is much higher than for the rare, highly luminous quasars found by, e.g., the Sloan Digital Sky Survey (e.g., Cristiani et al. 2004). Moderate-luminosity AGN at $z \approx 4-6$ can be selected effectively via the application of optical/near-infrared "dropout" techniques to X-ray sources. Without complementary X-ray data, many such AGN in the JDEM data will be difficult and observationally expensive to identify as they often have only modest AGN signatures in the optical/near-infrared. X-ray stacking techniques could also be applied to the tens of thousands of $z \approx 4-6 J D E M$-discovered galaxies that are not detected individually in X-rays. Such stacking would tightly constrain the frequency of low-luminosity AGN as well as X-ray emission associated with star formation.

Near-infrared JDEM observations of X-ray sources should also break the current AGN redshift "barrier" at $z \approx 6.5$, allowing the discovery of AGN out to $z \sim 10$. A small number of $z>6.5$ candidates have already been identified

in current deep X-ray surveys as extreme X-ray-to-optical ratio sources, but most and perhaps all probably lie at lower redshifts (Koekemoer et al. 2004). Wide fields with sensitive JDEM and X-ray coverage would hopefully gener- 

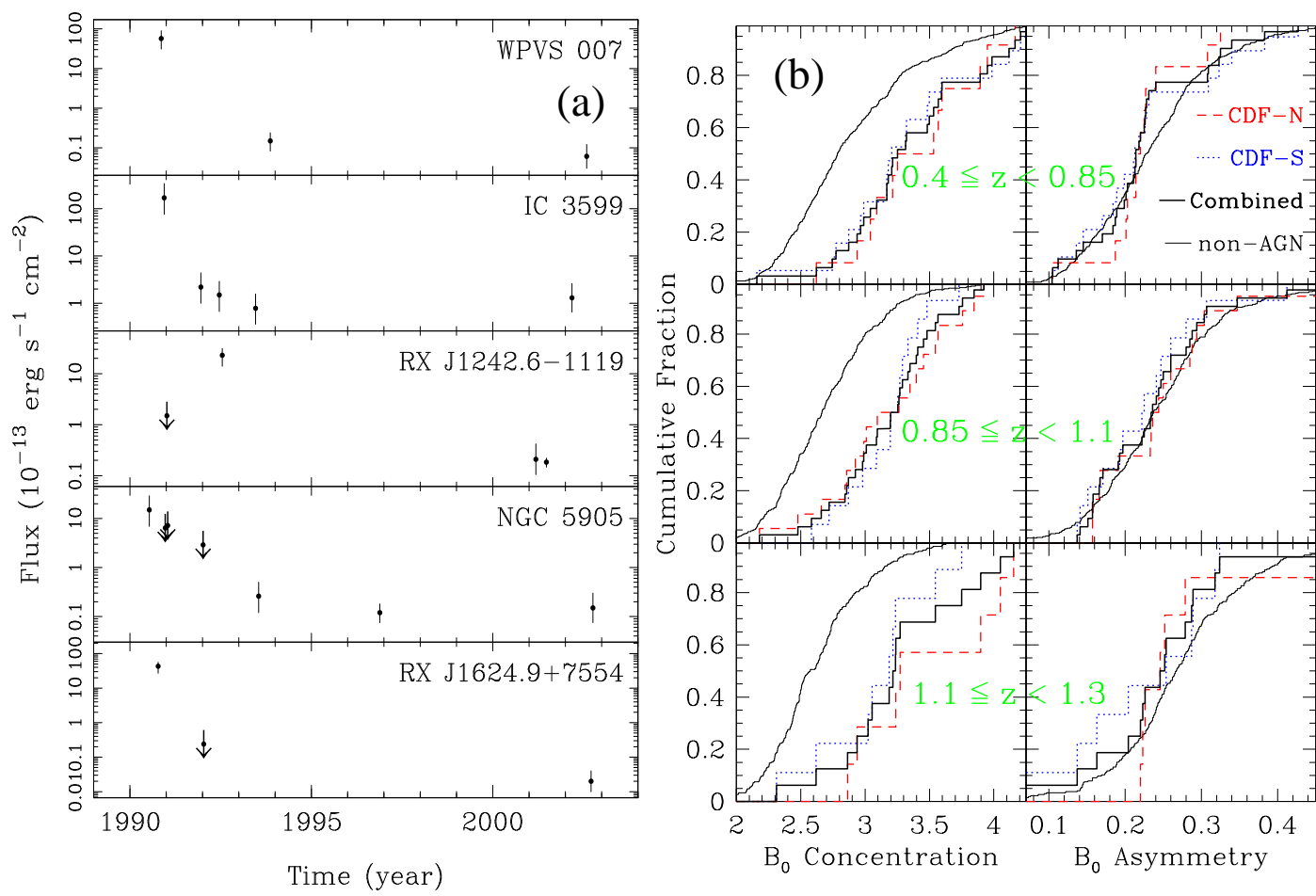

Fig. 3. (a) Historical X-ray light curves (from 0.3-2 keV) of five large-amplitude X-ray outbursts from galactic nuclei. WPVS 007, IC 3599, and NGC 5905 appear to be active galaxies, while the other two appear inactive. From Vaughan et al. (2004).

(b) Cumulative distribution functions of rest-frame $B$-band concentration index and asymmetry index for GOODS AGN hosts from the CDF-N and CDF-S. Cumulative distribution functions for normal field galaxies are also shown for comparison. Note that AGN hosts have more concentrated light profiles than normal field galaxies, but that there is no significant difference in asymmetry. From Grogin et al. (2004).

ate enough $z \approx 6.5-10$ AGN to elucidate how the black holes formed by the deaths of the first stars grew to make luminous quasars at $z \approx 4-6$.

\section{The Host-Galaxy Morphologies of X-ray Selected AGN}

High-resolution optical and near-infrared imaging, such as that to be provided by $J D E M$, is required to mitigate light blending between many moderateluminosity AGN and their host galaxies, thereby allowing the hosts to be studied reliably (see $\$ 1$ ). Grogin et al. (2004) have recently studied the host galaxies of samples of 100-200 moderate-luminosity, X-ray selected AGN at $z=0.4-1.3$ using HST imaging from the Great Observatories Origins Deep Survey (GOODS). They find that these AGN are preferentially hosted by galaxies with concentrated light profiles, generally corresponding to more bulge-dominated morphologies (see Fig. 3b). Similar results have been derived 
from Sersic-model fitting by B.D. Simmons et al., in preparation. Grogin et al. (2004) suggest that the locally observed relations between SMBH mass and host-galaxy properties are already in place at $z \approx 1$ (see, e.g., Graham et al. 2001 for the local relation between SMBH mass and concentration). JDEM imaging would allow these results to be refined using much larger samples, so that AGN of closely matched luminosity and other properties could be studied in fine redshift bins. Furthermore, the near-infrared capabilities of $J D E M$ would allow investigation of $\mathrm{SMBH} /$ host-galaxy relations at $z \gtrsim 1.3$, provided sufficient near-infrared sensitivity is achieved to overcome surfacebrightness dimming. The discovery of an epoch beyond which SMBHs and their host galaxies lose their tight relations would help to constrain models of $\mathrm{SMBH} /$ galaxy co-evolution.

Grogin et al. (2004) also investigated if their moderate-luminosity AGN samples showed enhanced merging or interaction activity relative to normal field galaxies, using an asymmetry index and near-neighbor counts (see Fig. 3b). They found no evidence for enhanced merging or interactions, results that are broadly consistent with those found for small samples of more luminous quasars (see, e.g., $\S 7$ of Dunlop et al. 2003). Wide fields surveyed by both $J D E M$ and X-ray missions would provide much larger AGN samples with good source statistics spanning a range of at least 10,000 in nuclear luminosity. More subtle differences in merging and interaction activity might then become statistically detectable, and differences could be searched for sensitively in subsets of the AGN population.

\section{Some General Prospects for X-ray Support of JDEM}

There are substantial challenges to obtaining wide-field X-ray data with sufficient sensitivity to complement JDEM properly. The exquisitely sensitive imaging of $J D E M$ will routinely reach $I=27-30$. To obtain reliable X-ray counterparts to optical sources at these faint flux levels, sub-arcsecond X-ray positions are required. Given the X-ray-to-optical flux ratios observed for X-ray selected sources (see Fig. 1), X-ray sensitivities of $\approx 10^{-17}-10^{-16} \mathrm{erg} \mathrm{cm}^{-2} \mathrm{~s}^{-1}$ (from $0.5-2 \mathrm{keV}$ ) are required to complement JDEM naturally. ${ }^{2}$ Such sensitivities correspond to Chandra exposures of 0.2-2 Ms. Thus, the total Chandra exposure required to cover the $15 \mathrm{deg}^{2} J D E M$ deep survey area appropriately

$\overline{2}$ Note that some superb high-energy survey missions currently being planned, such as DUO and EXIST, target comparatively bright X-ray flux levels that will not naturally match the sensitive optical/near-infrared imaging of JDEM. Most of the sources found by these high-energy survey missions are expected to have counterparts with $I=18-23$. JDEM will typically reach flux levels $\sim 1500$ times fainter than these counterparts. 
would be $\approx 1-10 \mathrm{yr} ;$ the wide-field $\left(\approx 500 \mathrm{deg}^{2}\right)$ and panoramic $\left(\approx 8000 \mathrm{deg}^{2}\right)$ JDEM surveys would require much more Chandra exposure. Even if Chandra survives until the launch of JDEM in $\approx 2014$, Chandra time-allocation committees may be hesitant to allocate the requisite enormous exposure!

Future X-ray missions with larger photon collecting areas coupled with excellent angular resolution, such as ESA's XEUS and NASA's Generation-X, offer better prospects for complementing JDEM effectively. The nominal launch date for $X E U S$ of $\approx 2015$ is fairly well synchronized with that of $J D E M$, and $X E U S$ could obtain appropriate coverage of the JDEM deep survey area in $\approx 0.1-1$ yr. Generation- $X$, currently under study as a NASA Space Science Vision Mission, would probably not begin operation until after $\approx 2020$. It could appropriately survey the JDEM deep survey area in about a week and could also complement the JDEM wide-field and panoramic surveys.

\section{Acknowledgments}

I thank D.M. Alexander, F.E. Bauer, N.A. Grogin, A.M. Koekemoer, B.D. Lehmer, V.L. Sarajedini, B.D. Simmons, A.T. Steffen, C.M. Urry, and C. Vignali for helpful discussions. Funding from NSF CAREER award AST-9983783 and CXC grant GO2-3187A is gratefully acknowledged.

\section{References}

[1] D.M. Alexander, F.E. Bauer, W.N. Brandt, et al., The Chandra Deep FieldNorth Survey. XIII. 2 Ms Point-Source Catalogs, AJ, 126 (2003) 539-574.

[2] F.E. Bauer, D.M. Alexander, W.N. Brandt, et al., The Fall of AGN and the Rise of Star-Forming Galaxies: A Close Look at the Chandra Deep Field X-ray Number Counts, $A J$, in press.

[3] W.N. Brandt, K.A. Pounds, and H. Fink, The Unusual X-ray and Optical Properties of the Ultrasoft Active Galactic Nucleus Zwicky 159.034 (RE J1237+264), MNRAS, 273 (1995) 47-52.

[4] M. Cappellari, A. Renzini, L. Greggio, et al., The Mini-Active Galactic Nucleus at the Center of the Elliptical Galaxy NGC 4552 with Hubble Space Telescope, ApJ, 519 (1999) 117-133.

[5] S. Cristiani, D.M. Alexander, F.E. Bauer, et al., The Space Density of HighRedshift QSOs in the Great Observatories Origins Deep Survey, ApJ, 600 (2004) L119-L122. 
[6] G. de Vaucouleurs, Flare-up in the nucleus of NGC 1068 in 1980?, The Observatory, 111 (1991) 122-123.

[7] J.L. Donley, W.N. Brandt, M. Eracleous, and Th. Boller, Large-Amplitude X-ray Outbursts from Galactic Nuclei: A Systematic Survey Using ROSAT Archival Data, AJ, 124 (2002) 1308-1321.

[8] J.S. Dunlop, R.J. McLure, M.J. Kukula, et al., Quasars, Their Host Galaxies and Their Central Black Holes, MNRAS, 340 (2003) 1095-1135.

[9] A.W. Graham, P. Erwin, N. Caon, and I. Trujillo, A Correlation Between Galaxy Light Concentration and Supermassive Black Hole Mass, ApJ, 563 (2001) L11L14.

[10] N.A. Grogin, C.J. Conselice, E. Chatzichristou, et al., GOODS AGN Host Structural Parameters and Environment: Evidence for Black Hole-Bulge Correlation and Against Merger-AGN Connection at $z \sim 0.4-1.3, A p J$, submitted.

[11] D. Grupe, K. Beuermann, K. Mannheim, et al., X-ray Outburst of the Peculiar Seyfert Galaxy IC 3599, A\&A, 299 (1995) L5-L8.

[12] A. Koekemoer, D.M. Alexander, F.E. Bauer, et al., A Possible New Population of Sources with Extreme X-ray/Optical Ratios, ApJ, 600 (2004) L123-L126.

[13] S. Komossa, X-ray Evidence for Supermassive Black Holes at the Centers of Nearby, Non-Active Galaxies, Rev. Mod. Ast., 15 (2002) 27-56.

[14] V.L. Sarajedini, R.L. Gilliland, and C. Kasm, A $V$-Band Survey for Variable Galactic Nuclei in the Hubble Deep Field, ApJ, 599 (2003) 173-184.

[15] S. Vaughan, R. Edelson, and R.S. Warwick, Chandra Observations of Five X-ray Transient Galactic Nuclei, MNRAS, 349 (2004) L1-L5. 